\title{
Fertility awareness methods of family planning
}

1. The woman's egg lives up to 7 days. False

Answer

2. Sperm can survive in the female genital tract and are capable of fertilising the egg for up to 7 days after sex. True

3. The chances of conception are approximately zero six days before and 2 days after the temperature rise. True

4. The basal body temperature rises by about $0.5^{\circ} \mathrm{C}$ just before ovulation.

False

5. Usually when a woman is fertile her cervix is high, soft and open.

True

6. To optimise her chances of pregnancy a woman should be aware of the significance of timing intercourse to coincide with wetter, transparent, slippery, stretchy secretions.

True

7. The late infertile time (post-ovulation) is the safest time for intercourse to avoid pregnancy.

True

8. The standard days rule indicates the fertile time as days 10-23 for all women (provided cycles are within the range of 26-32 days).

False

9. When motivated couples taught by experienced FAM teachers use a combination of fertility indicators, fertility awareness methods can be up to $98 \%$ effective.

True

10. Lactational amenorrhoea method only applies to women who are less than 6 months post-partum, amenorrhoeic and fully breastfeeding day and night.

True 